\title{
Lense-Thirring Precession and QPOs in Low Mass X-Ray Binaries
}

\author{
Luigi Stella ${ }^{1,3}$ and Mario Vietri ${ }^{2}$
}

\begin{abstract}
We show in this Letter that relativistic dragging of inertial frames around fast rotating collapsed stars is substantial and can give rise to observable effects. We apply this to the $\mathrm{kHz}$ quasi periodic oscillations (QPOs) sources, low mass X-ray binaries (LMXRBs) containing an accreting neutron star. Within the beat frequency model, both the Keplerian frequency of the innermost region of the accretion disk $(\sim 0.3-1.2 \mathrm{kHz})$ and the neutron star spin frequency $(\sim 0.3-0.4 \mathrm{kHz})$ are directly observed. From these the Lense-Thirring precession frequency (tens of $\mathrm{Hz}$ ) of the same material in the innermost disk regions which gives rise to the $\mathrm{kHz}$ QPOs is determined within a factor of $\sim 4$, depending on the neutron star equation of state. The classical contribution from neutron star oblateness decreases the precession frequency slightly. The broad peaks at frequencies $\sim 20-40 \mathrm{~Hz}$ in the power spectra of the "Atoll"-sources 4U 1728-34, 4U 0614+091 and KS 1731-260 and their variations with the higher $\mathrm{kHz}$ QPO frequency are well matched by Lense-Thirring precession of material in the innermost disk region. We also suggest that the $\sim 15-50 \mathrm{~Hz}$ horizontal branch QPOs of GX 5-1 and GX 17+2 (and likewise other "Z"-type low mass X-ray binaries) arise from the same mechanism.
\end{abstract}

Subject headings: accretion, accretion disks — relativity — pulsars: general — stars: neutron, rotation $-\mathrm{X}$-rays: stars

\section{Introduction}

The variety of QPO modes from old accreting neutron stars in low mass X-ray binaries (LMXRBs) (see e.g. van der Klis 1995) has been recently enriched by the discovery of kiloHertz oscillations in light curves obtained with the Rossi X-ray Timing Explorer (RXTE). These are the first QPOs that involve timescales comparable to the dynamical timescales in the vicinity of a collapsed star. There are currently eleven LMXRBs from which QPOs have been detected between frequencies of $\sim 0.3$ and $1.2 \mathrm{kHz}$.

Most of these are type I X-ray bursters with persistent luminosities in the $10^{36}-10^{37} \mathrm{ergs} \mathrm{s}^{-1}$ range, that according to the classification of Hasinger and van der Klis (1989) either belong to (4U 1728-34, 4U 1608-52, $4 \mathrm{U} 1636-536,4 \mathrm{U} 1735-444$ and $4 \mathrm{U} 1820-30)$ or are suspected members of (4U 0614+091 and KS 1731-260) the "Atoll" group. Sco X-1, GX 5-1 and GX 17+2 are instead high luminosity "Z"-type LMXRBs.

A pair of $\mathrm{kHz}$ QPO peaks is often present in the power spectra. This is the case for the persistent emission of the five Atoll sources, at least on occasions and/or over some range of count rates. In $4 \mathrm{U} 1728-34$ and $4 \mathrm{U} 0614+091$ the centroid frequency of the QPOs is positively correlated with the source count rate across the factor of $\sim 2$ QPO frequency variations (from $\sim 500$ to $\sim 1100 \mathrm{~Hz}$ ) observed from both sources. When a pair of peaks is present, the difference $\Delta \nu$ between their centroid frequencies $\nu_{1}$ and $\nu_{2}$ is consistent with being constant. In $4 \mathrm{U} 1728-34 \Delta \nu$ remains around $\simeq 363 \mathrm{~Hz}$ (within a few percent) as the frequency of the $\mathrm{kHz}$ QPOs changes by $\sim 20 \%$. A nearly coherent signal at $\sim 363 \mathrm{~Hz}$ has been detected during a number of X-ray

\footnotetext{
${ }^{1}$ Osservatorio Astronomico di Roma, Via dell'Osservatorio 2, 00040 Monte Porzio Catone (Roma), Italy, e-mail: stella@coma.mporzio.astro.it

${ }^{2}$ Università di Roma 3, Via della Vasca Navale 84, 00147 Roma, Italy, e-mail: vietri@corelli.fis.uniroma3.it

${ }^{3}$ Affiliated to the International Center for Relativistic Astrophysics
} 
bursts (Strohmayer et al. 1996a). In the persistent emission of $4 \mathrm{U} 0614+091$ the separation $\Delta \nu$ is consistent with $\sim 323 \mathrm{~Hz}$ (within $\sim 10 \%$ ), across a $\sim 50 \%$ variation of the QPO frequency and a $\sim 100 \mathrm{~d}$ interval between two RXTE observations. During part of an observation, a third narrower peak has been marginally detected at a frequency of $\sim 328 \mathrm{~Hz}$, consistent with the separation of the pair of $\mathrm{kHz}$ QPO peaks (Ford et al. 1997). In the persistent emission of KS 1731-260, two kHz QPO peaks have been detected around $\sim 900$ and $\sim 1160 \mathrm{~Hz}$. The frequency difference $(\sim 260 \mathrm{~Hz})$ between these QPOs is consistent with half the frequency of the nearly coherent $524 \mathrm{~Hz}$ signal observed in a type I burst from this source (Smith et al. 1997; Wijnands \& van der Klis 1997). kHz QPOs have also been discovered in $4 \mathrm{U}$ 1608-52 (Berger et al. 1996) and 4U 1735-444 (Wijnands et al. 1996).

A QPO peak with an intensity-dependent frequency from $\sim 550$ to $\sim 800 \mathrm{~Hz}$ has been detected in $4 \mathrm{U}$ 1820-30. Close to the upper end of this range a second QPO peak at $\sim 1070 \mathrm{~Hz}$ is present (Smale, Zhang \& White 1997). In the persistent emission of $4 \mathrm{U} 1636-536$ a pair of QPO peaks around 840-920 $\mathrm{Hz}$ and 1150-1200 $\mathrm{Hz}$ have been detected, the frequency separation of which is constant around $\Delta \nu \sim 255 \pm 25 \mathrm{~Hz}$. A nearly coherent signal at $\sim 581 \mathrm{~Hz}$ modulates the X-ray flux of this source during type I bursts (Zhang et al.1996, Wijnands et al.1997); note that half this frequency is compatible with the separation $\Delta \nu$. Periodic oscillations at $589 \mathrm{~Hz}$ have also been observed during type I bursts from an unknown burster in the direction of the galactic centre, perhaps MXB 1743-29 (Strohmayer et al. 1996b).

GX 5-1, GX 17+2 and Sco X-1, currently the only high-luminosity "Z"-type LMXRBs in the group, display $\mathrm{kHz}$ QPOs $(\sim 0.3-1.1 \mathrm{kHz})$ with a centroid frequency which is positively correlated with the inferred mass accretion rate. In GX 5-1 a pair of $\mathrm{kHz}$ QPO peaks has been detected over part of the horizontal branch (HB), which mantain a constant separation of $\Delta \nu \simeq 325 \mathrm{~Hz}$. The higher frequency QPO peak of GX 5-1 increases from $\nu_{2} \sim 570$ to $\sim 900 \mathrm{~Hz}$ as the source moves from the left to the right end of the $\mathrm{HB}$ (van der Klis et al. 1996a). In GX $17+2$ a kHz QPO peak is observed to drift from $\sim 680$ to $880 \mathrm{~Hz}$ QPO as the source moves down the upper part of the so-called normal branch. A higher frequency peak separated by $\sim 306 \mathrm{~Hz}$ is observed close to the lower end of this range, together with an additional QPO peak around $\sim 60 \mathrm{~Hz}$, interpreted as HB oscillations (van der Klis et al. 1997a). In Sco X-1, $\sim 0.5-1.1 \mathrm{kHz}$ QPOs have been detected in the normal and flaring branches; their frequency correlates well both with the inferred variations of the mass accretion rate and the frequency variations of the $\sim 6-20 \mathrm{~Hz}$ QPO that occur along the two branches. A pair of $\mathrm{kHz}$ QPO peaks has been detected on occasions. However their separation decreased from $\Delta \nu \sim 310$ to $\sim 230 \mathrm{~Hz}$ as the higher frequency peak moved from $\sim 875$ to $\sim 1085 \mathrm{~Hz}$. Correspondingly additional QPOs at $\sim 40-50 \mathrm{~Hz}$ were present, which are intepreted as HB QPOs (van der Klis et al. 1996b, 1997b).

Modelling of $\mathrm{kHz}$ QPOs has focussed on the presence of peaks at $\nu_{1}$ and $\nu_{2}$, the constancy of their separation $\Delta \nu$ and the coherence of the signal (either during type I bursts or in the persistent emission) at frequency $\Delta \nu($ or $2 \Delta \nu)$ 周.

The application of beat frequency concepts to these signals is natural. In the magnetospheric beat frequency model, MBFM, originally developed to interpret the 5-50 Hz QPOs from high luminosity "Z"-type LMXRBs (Alpar \& Shaham 1985; Lamb et al. 1985), matter inhomogeneities in the accretion disk cause accretion to be modulated at the beat frequency between the Keplerian frequency, $\nu_{K}$, of the innermost region of the accretion disk $\left(r_{i n}\right)$ at the magnetospheric boundary, and the neutron star spin frequency, $\nu_{s}$. As $r_{i n}$ decreases with increasing accretion rates, $\nu_{K}$ increases and so does the QPO beat frequency $\nu_{b}=\nu_{K}-\nu_{s}$. Of the various QPO modes with frequencies $<100 \mathrm{~Hz}$ in "Z"-type LMXRBs, only HB QPOs are currently interpreted in terms of the MBFM (for a review see Lamb 1991). The basic physical picture of the MBFM has been confirmed mainly through the study of QPOs in a few accreting X-ray pulsars (EXO 2030+375 and A $0535+26$ in particular), where, unlike "Z"-type LMXRBs, the neutron star spin frequency $\nu_{s}$ is (by definition) clearly detected, and independent estimates of the neutron star magnetic field exist (Angelini et al. 1989; Finger et al. 1996).

\footnotetext{
4 Though these properties are not observed in all LMXRBs with kHz QPOs, they appear to be common, with Sco X-1 the only clear exception to the constancy of $\Delta \nu$ (White \& Zhang 1997)
} 
Besides the signals at $\nu_{s}$ and $\nu_{b}$, an additional QPO signal at $\nu_{K}$ is expected in the MBFM from inhomogeneities at the inner disk boundary. For self-luminous blobs, the amplitude of this signal should be higher for smaller values of $r_{i n}$, due to the larger local gravitational energy and Keplerian velocities (causing a larger Doppler boosting around the orbit). Similarly, a small value of $r_{i n}$ increases the range of inclination angles for which a signal at $\nu_{K}$ can be caused by the neutron star occulting the blobs at $r_{i n}$ or conversely, by the blobs at $r_{i n}$ obscuring part of the radiation produced closer to the neutron star surface.

In the MBFM for $\mathrm{kHz}$ QPOs from LMXRBs, the lower frequency QPOs are interpreted as the beat frequency $\left(\nu_{1} \simeq \nu_{b}\right)$ and the higher frequency QPOs as the Keplerian frequency $\left(\nu_{2} \simeq \nu_{K}\right)$ at the magnetospheric boundary. The nearly coherent signal, occasionally detected at a frequency compatible with the separation $\Delta \nu$ of the QPO pair (or with $2 \Delta \nu$ ), provides the first direct evidence of the neutron star spin frequency $\nu_{s}$ in LMXRBs containing an old neutron star (Strohmayer et al. 1996a; Ford et al. 1997). Inferred magnetospheric radii $r_{m} \simeq r_{i n}=(G M)^{1 / 3}\left(2 \pi \nu_{K}\right)^{-2 / 3}=15 M_{0}^{1 / 3} \nu_{K 3}^{-2 / 3} \mathrm{~km}$, (here $M=M_{0} \mathrm{M}_{\odot}$ is the neutron star mass and $\nu_{K}=10^{3} \nu_{K 3} \mathrm{~Hz}$ ) correspond to a couple of neutron star radii at the most. The neutron star spin frequency of several hundred $\mathrm{Hz}$ and the inferred magnetic field strength of $\sim 10^{8}-10^{9} \mathrm{G}$ agree with spin-up by accretion torques, and scenarios for the formation of millisecond pulsars.

Miller et al. (1996) proposed an alternative beat frequency model based on the idea that the magnetic field of the neutron star is so low that it does not affect the dynamics of the innermost disk regions and, yet, high enough to give rise to beamed radiation at the neutron star surface. We do not discuss the details of this model, but notice that here too the QPO signal at the highest frequency $\nu_{2}$ corresponds to the innermost Keplerian frequency of the disk, which in this model ends at the sonic radius where the radial velocity becomes supersonic (as opposed to the magnetospheric radius), and the lower frequency $\nu_{1}$ is the beat frequency between the Keplerian frequency and the neutron star spin frequency, exactly like in the MBFM.

\section{Lense-Thirring Precession in LMXRBs with kHz QPO}

The parameters inferred from beat frequency models for the $\mathrm{kHz}$ QPO from LMXRBs are such that frame dragging, one of the general relativistic effects of the "gravitomagnetic" field associated with rotating bodies, is substantial and can lead to observable phenomena. In particular the orbital plane of a test particle, if not coaligned with the equatorial plane, must precess in a prograde fashion around the angular momentum axis of the rotating object. In the weak field limit the nodal precession frequency, first calculated by Lense \& Thirring (1918) (see also Wilkins 1972), is given by $\nu_{L T}=G M a /\left(\pi c^{2} r^{3}\right)$, where $M$ and $a$ are the mass and specific angular momentum of the rotating object and $r$ is the radius of the test particle (circular) orbit. By using $M a=2 \pi I \nu_{s}$, and $r=(G M)^{1 / 3} /\left(2 \pi \nu_{K}\right)^{2 / 3}$ this can be written as

$$
\nu_{L T}=\frac{8 \pi^{2} I \nu_{K}^{2} \nu_{s}}{c^{2} M}=13.2 I_{45} M_{0}^{-1} \nu_{K 3}^{2} \nu_{s 2.5} \quad \mathrm{~Hz}
$$

where $I=10^{45} I_{45} \mathrm{~g} \mathrm{~cm}^{2}$ is the moment of inertia of the neutron star and $\nu_{s}=300 \nu_{s 2.5} \mathrm{~Hz}$ its spin frequency. By using the values of $\nu_{s}$ and $\nu_{K}$ inferred from the application of beat frequency models to the $\mathrm{kHz}$ QPOs from LMXRBs, it is apparent that Lense-Thirring frequencies of a few tens of $\mathrm{Hz}$ are expected in the innermost disk region which produces the QPO signal at $\nu_{K}$. Note also that in this regime, higher order terms in $a / M$ would provide a correction to Eq. (1) at a level of less than one percent.

Since sources with a pair of $\mathrm{kHz}$ QPO peaks allow identification of both $\nu_{K}$ and $\nu_{s}$, the only parameter in Eq. (1) that is not immediately identified from observations is $I / M$. The theoretical uncertainty in its determination depends on the choice of the equation of state (EOS) and the star mass: from models of rotating neutron stars (Friedman, Ipser and Parker 1986; Cook, Shapiro \& Teukolsky 1994), it can be seen that $I / M$ varies in the range $0.5<I_{45} / M_{0}<2$ for any mass and EOS. We notice that $\nu_{L T}$ in Eq. (1) is thus independent of any other uncertainty related to LMXRBs, such as source distance, mass accretion rate or efficiency of conversion of gravitational energy into radiative energy. 
The neutron star spin frequencies discussed here lead to a stellar oblateness which, in turn, causes the appearance of a quadrupole term in the gravitational potential. It is well-known that the quadrupole term causes a precession of orbits tilted away from the star equatorial plane, with the precession frequency $\propto r^{-7 / 2}$ (as opposed to $r^{-3}$ for the Lense-Thirring precession). For the small radii of interest to us, it is important to check that this classical precession rate is still smaller than the Lense-Thirring rate, to make sure that the effect we predict is a genuine relativistic one. The classical precession frequency $\nu_{c l}$ is given by (Kahn and Woltjer 1959)

$$
\nu_{c l}=\frac{3}{8 \pi^{2}} \frac{\phi_{2} \cos \beta}{r^{2} \nu_{K}},
$$

where $\beta$ is the tilt angle off the equatorial plane, and $\phi_{2}$ is the coefficient of the quadrupole term in an expansion of the gravitational potential in spherical harmonics, $\phi \approx G M / r+\phi_{2} P_{2}(\cos \theta)$, with $P_{2}$ the second order Legendre polynomial. Outside the star, we have $\phi_{2}=G A / r^{3}$, where the coefficient $A$ is given, in terms of the star moment of inertia, by $A=\left(I_{x x}+I_{y y}-2 I_{z z}\right) / 2$, where $z$ is the star rotation axis. For rotationally flattened stars, far from breakup, $I_{x x}=I_{y y}$. For rotationally flattened stars, $A<0$, and both $I_{x x}$ and $I_{z z}$ are increased by rotation. In the existing literature we have been able to find only the numerical values of $I_{z z}^{N R}$ for non-rotating neutron star models and the corresponding rotation-increased values, $I_{z z}^{R}$ (Friedman, Ipser and Parker 1986, Cook, Shapiro and Teukolsky 1994). Using the rotating polytropic models calculated by James (1964), we derived the approximate relation $I_{z z}^{N R}-I_{z z}^{R} \simeq 2\left(I_{x x}^{R}-I_{z z}^{R}\right)$. Assuming that this holds also for neutron star models, we have $A \simeq\left(I_{z z}^{N R}-I_{z z}^{R}\right) / 2$. This is probably a fairly accurate approximation because the rotation frequencies we are interested in, $\nu_{s} \lesssim 500 \mathrm{~Hz}$, correspond to still slowly rotating neutron star models: in fact, perusal of the tabulated models in the references given above shows that the ratio $t$ of rotational to binding energy for these $\nu_{s}$ is $\approx 0.01-0.04$, while models nearly at breakup have $t z 0.11$, for all EOS. We find, defining $A \equiv-\eta \nu_{s 2.5}^{2} I$, where $I=I_{z z}^{N R}$, that the parameter $\eta$ is roughly independent of spin frequency in the slow-rotation regime of interest here, and it depends, albeit weakly, upon model mass and EOS (range of $\left.\eta \approx(0.5-3.2) \times 10^{-2}\right)$. Eventually, we find

$$
\nu_{c l}=-4.7 I_{45} M_{0}^{-5 / 3}\left(\frac{\eta}{10^{-2}}\right) \cos \beta \nu_{K 3}^{7 / 3} \nu_{s 2.5}^{2} H z .
$$

Note that the negative sign reflects the fact that classical precession is retrograde for $\beta<\pi / 2$. Comparison of this with equation 1 shows that, even at these small radii, Lense-Thirring precession is faster. The total precession frequency $\nu_{p}=\nu_{L T}+\nu_{c l}$ is thus somewhat decreased by oblateness-induced precession.

Modulation of the X-ray flux at the precession frequency of the inner disk boundary is expected to occur through the same geometrical effects producing the modulation at the Keplerian frequency. In fact, the precessional motion of the blobs generating the signal at $\nu_{K}$ would give rise to a signal at the precession frequency, both for self-luminous blobs (the projected velocity along the line of sight and, therefore, the Doppler boosting will be modulated at the precession frequency) and occulting, or occulted, blobs (the duration of the occultations wiil depend on the phase of the precessional motion). Moreover we note that, unlike the signal at $\nu_{K}$, an X-ray modulation at the precession frequency can in principle be generated, even in the absence of blobs, by the changing aspect of the innermost disk ring. This would modulate the ring emission as seen from the earth or could partially occult the emitting regions close to the neutron star surface.

Lense-Thirring precession of viscous accretion disks is generally neglected because, following the pioneering work by Bardeen and Patterson (1975), many authors found that viscosity leads the disk to lie in the equatorial plane of the rotating object. However, this conclusion has recently been challenged by (some of) the same authors: even if the innermost disk regions are confined to the equatorial plane of the neutron star, Pringle (1996) and Maloney, Begelman and Pringle (1996) have shown that radiation pressure can lead to corrugation of the disk and its lifting off the neutron star equatorial plane. Pringle (1996) has shown that even initially planar disks are unstable to warping, so that tilting should be generic in centrally illuminated sources. Precessing disks have also been recently revisited by Papaloizou et al.(1997). It is possible that other effects, such as parametric amplification (Binney 1978) of the $\mathrm{z}$-oscillations of diamagnetic blobs by a dipolar magnetic field tilted with respect to the star spin axis, may intervene (Vietri and Stella, in preparation).

The power spectra of 4U 1728-34 given in Fig. 1a clearly show a broad peak centered around a frequency 
of $\sim 20,26$ and $35 \mathrm{~Hz}$ when the higher frequency QPO peak is at a frequency of $\nu_{2} \sim 900,980$ and $1100 \mathrm{~Hz}$, respectively. The width of the $20-35 \mathrm{~Hz}$ peak is of $\sim 15-20 \mathrm{~Hz}$. The power spectrum of $4 \mathrm{U} 0614+091$ (see Fig. 1b) and KS 1731-260 (see Fig. 1c) also shows a similarly broad peak centered around $\sim 22 \mathrm{~Hz}$ and $\sim 27 \mathrm{~Hz}$, when $\nu_{2} \sim 900 \mathrm{~Hz}$ and $\nu_{2} \sim 1200 \mathrm{~Hz}$, respectively. We propose that these tens of $\mathrm{Hz}$ QPO peaks arise from the precession of the innermost disk regions, where the signals at the Keplerian and beat frequencies also originate. The thick bars in Fig. 1 represent the precession frequency calculated from the sum of the Lense-Thirring and classical frequencies (Eqs. 1 and 3) by using $\nu_{K}=\nu_{2}$ (see above), and $\nu_{s}=363,323$ and $262 \mathrm{~Hz}$ in the case of $4 \mathrm{U} 1728-34,4 \mathrm{U} 0614+091$ and KS 1731-260, respectively. In all three cases, we adopted the same neutron star model with EOS L, $M_{0}=1.97, I_{45} / M_{0}=1.98$ and $\eta=10^{-2}$ (Friedman, Ipser \& Parker 1986). Lense-Thirring precession dominates, with the classical term providing a $\sim 20 \%$ correction. The fact that the (absolute) width of the tens of $\mathrm{Hz}$ QPO peaks is comparable to that of the $\mathrm{kHz}$ QPO peaks indicates that lifetime broadening dominates the coherence in both cases.

Both $\nu_{K}$ and $\nu_{s}$ are independently determined in these three sources. Thus the range of precession frequencies allowed by the model is determined only by the neutron star parameters $I_{45} / M_{0}$ and, to a lesser extent, $M_{0}$ and $\eta$. The intervals marked by the filled squares and thick bars in Fig. 1 represent the whole range of precession frequencies that has been obtained by using virtually all neutron star models given by Friedman, Ipser \& Parker (1986) and Cook, Shapiro \& Teukolsky (1994) (we excluded models with $M_{0} \sim 0.5$ and "supramassive" models). We regard the very good agreement with the frequency of the corresponding power spectrum peaks as a strong indication in favor of our relativistic plus classical precession interpretation. We note that for all three sources the QPO peaks are very close to the high-frequency end of the intervals corresponding to the relatively stiff EOS L and $M_{0} \sim 2$. As the frequency of the $\mathrm{kHz}$ QPOs from $4 \mathrm{U} 1728-34$ varied, the best fit precession frequency remained in good agreement with the observed peak, showing that the power law dependence (Eq. 1) is probably in the correct range.

The detection of $\mathrm{kHz}$ QPOs in the HB of GX 5-1 is especially important for the modelling of QPOs in "Z"-type LMXRBs. If one retains the standard interpretation in which the $15-50 \mathrm{~Hz}$ QPOs in the HB arise from the MBFM, then a magnetosphere extending up to $>5$ neutron star radii is required (implying a magnetic field of $\sim 10^{9} \mathrm{G}$ ) which argues against any interpretation of the $\mathrm{kHz}$ QPOs involving Keplerian orbits at $\sim 15-20 \mathrm{~km}$. If on the contrary, a Keplerian beat frequency model is adopted for the $\mathrm{kHz}$ QPOs, then the $15-50 \mathrm{~Hz}$ QPOs are unlikely to arise from the MBFM. We regard the presence of a pair of $\mathrm{kHz}$ QPOs and the constancy of their frequency difference as strong evidence in favor of the latter interpretation. In this context, it appears natural to interpret also the HB QPOs of GX 5-1 in terms of our precession model. For $\nu_{s}=\Delta \nu \sim 325 \mathrm{~Hz}$ and $\nu_{K}=\nu_{2} \sim 570-900 \mathrm{~Hz}$, the maximum precession frequency (again the one from EOS $\mathrm{L}$ and $M_{0} \simeq 2$ ) is $\sim 8-20 \mathrm{~Hz}$. Similarly in the case of GX $17+2$, we obtain a maximum precession frequency of $\sim 22 \mathrm{~Hz}$, when the source is in the upper normal branch with $\nu_{K}=\nu_{2} \sim 988 \mathrm{~Hz}$ and $\nu_{s}=\Delta \nu \sim 306 \mathrm{~Hz}$, and $\mathrm{HB}$ QPOs at $\sim 60 \mathrm{~Hz}$ are simultaneously present. These precession frequencies are clearly a factor of 2-2.5 smaller than those of the HB QPOs. One possibility is that the neutron stars of "Z"-type LMXRBs have $I_{45} / M_{0} \sim 4-5$, i.e. a somewhat higher value than Friedman, Ipser \& Parker (1986) and Cook, Shapiro \& Teukolsky (1994) calculate. Alternatively the HB QPOs might arise from the second harmonics of the precession frequency, due to some yet unknown reason. We note that in the case of GX 5-1 the dependence of the HB QPO frequency on $\nu_{2}$ is in the same range as the dependence of $\nu_{p}$ on $\nu_{K}$ in our model.

In summary, we propose that the peaks observed in the power spectra of several "Atoll" LMXRBs at a frequency of tens of $\mathrm{Hz}$ (and perhaps also the HB QPOs of "Z"-type LMXRBs) are due to precession of the innermost disk region, which is dominated by the Lense-Thirring effect. The precession frequency depends upon the Keplerian frequency at the innermost disk radius, and the neutron star spin frequency, both of which are independently determined through the application of beat frequency models to the $\mathrm{kHz}$ QPOs from these sources. The best fit is obtained for a stiff EOS and $M_{0} \simeq 2$, a value supported also from the interpretation of the highest observed values of $\nu_{2}$ in terms of the Keplerian frequency of the marginally stable orbit (Kaaret, Ford \& Chen 1997; Zhang, Strohmayer \& Swank 1997).

LS acknowledges useful discussions with D. Bini, I. Ciufolini, D. Psaltis. This work was partially supported through ASI grants. 


\section{REFERENCES}

Alpar, A., \& Shaham, J. 1985, Nature 316, 239

Angelini, L., Stella, L. \& Parmar, A.N. 1989, ApJ 346, 906

Bardeen, J.M., Patterson, J.A., 1975, ApJ, 195, L65

Berger, M., et al., 1996, ApJ, 469, L13

Binney, J.J., 1978, MNRAS, 183, 779

Cook, G.B., Shapiro, S.L. \& Teukolsky, S. 1994, ApJ, 424, 823

Finger, M. H., Wilson, R. B. \& Harmon, B. A. 1996, ApJ, 459, 288

Ford, E., et al., 1997, ApJ, 475, L123

Friedman, J.L, Ipser, J.R. \& Parker, L. 1986, ApJ, 304, 115

Ghosh, P., \& Lamb, F.K. 1979, ApJ, 234, 296

Ghosh, P., \& Lamb, F.K. 1992, in X-ray Binaries and Recycled Pulsars, Ed. E.P.J. van den Heuvel and S.A. Rappaport (Dordrecht: Kluwer), p.487

Hasinger, G., \& van der Klis, M. 1989, A\&A 225, 79

James, R. A. 1964, ApJ, 140, 552

Kaaret, P., Ford, E.G. \& Chen, K. 1997, ApJL, 480, L27

Kahn, F.D. \& Woltjer L. 1959, ApJ, 130, 705

Lamb, F.K., 1991, in Neutron Stars: Theory and Observation, Ed. J. Ventura and D. Pines, (Dordrecht: Kluwer), p.445

Lamb, F.K., Shibazaki, N., Alpar, A., \& Shaham, J. 1985, Nature 317, 681

Lense, J., \& Thirring, H. 1918, Physik. Z. 19, 156

Maloney, P.R., Begelman, M,C., Pringle, J.E., 1996, ApJ, 472, 582

Miller, M.C., Lamb, F.K., \& Psaltis, D. 1997, ApJ, submitted

Papaloizou, J.C.B., Larwood, J.D., Nelson, R.P., Terquem, C., 1997, to appear in "Proceedings of the EARA Workshop on accretion disks", Lecture Notes in Physics.

Pringle, J.E., 1992, MNRAS, 258, 811

Pringle, J.E., 1996, MNRAS, 281, 357

Smale, A.P., Zhang, W., White, N.E., 1997, ApJL, 483, L119

Smith, D.A., Morgan, E.H. \& Bradt, H. 1997, ApJ, 479, L137

Strohmayer, T., et al., 1996a, ApJ 469, L9

Strohmayer, T., Lee, U., \& Jahoda, K. 1996b, IAU Circ. 6484

van der Klis, M. 1995, in X-ray Binaries, Eds. W. H. G. Lewin, J. van Paradijs \& E. P. J. van den Heuvel (Cambridge University Press), p. 252

van der Klis, M., et al., 1996a, IAU Circ. 6511 
van der Klis, M., et al., 1996b, ApJ, 469, L1

van der Klis, M., et al., 1997a, IAU Circ. 6565

van der Klis, M., Wijnands, R.A.D., Horne, K. \& Chen, W. 1997b, ApJL, 481, L97

White, N.E. \& Zhang, W. 1997, ApJL, submitted

Wijnands, R.A.D., et al., 1996, IAU Circ. 6447

Wijnands, R.A.D., et al., 1997, ApJL, 479, L141

Wijnands, R.A.D. \& van der Klis, M. 1997, ApJL, 482, L65

Wilkins, D.C. 1972, Phys. Rev. D 5, 814

Zhang, W., Lapidus, I., White, N.E., \& Titarchuk, L. 1996, ApJ, 469, L17

Zhang, W., Strohmayer, T.E. \& Swank, J.H. 1997, ApJL, 482, L167 

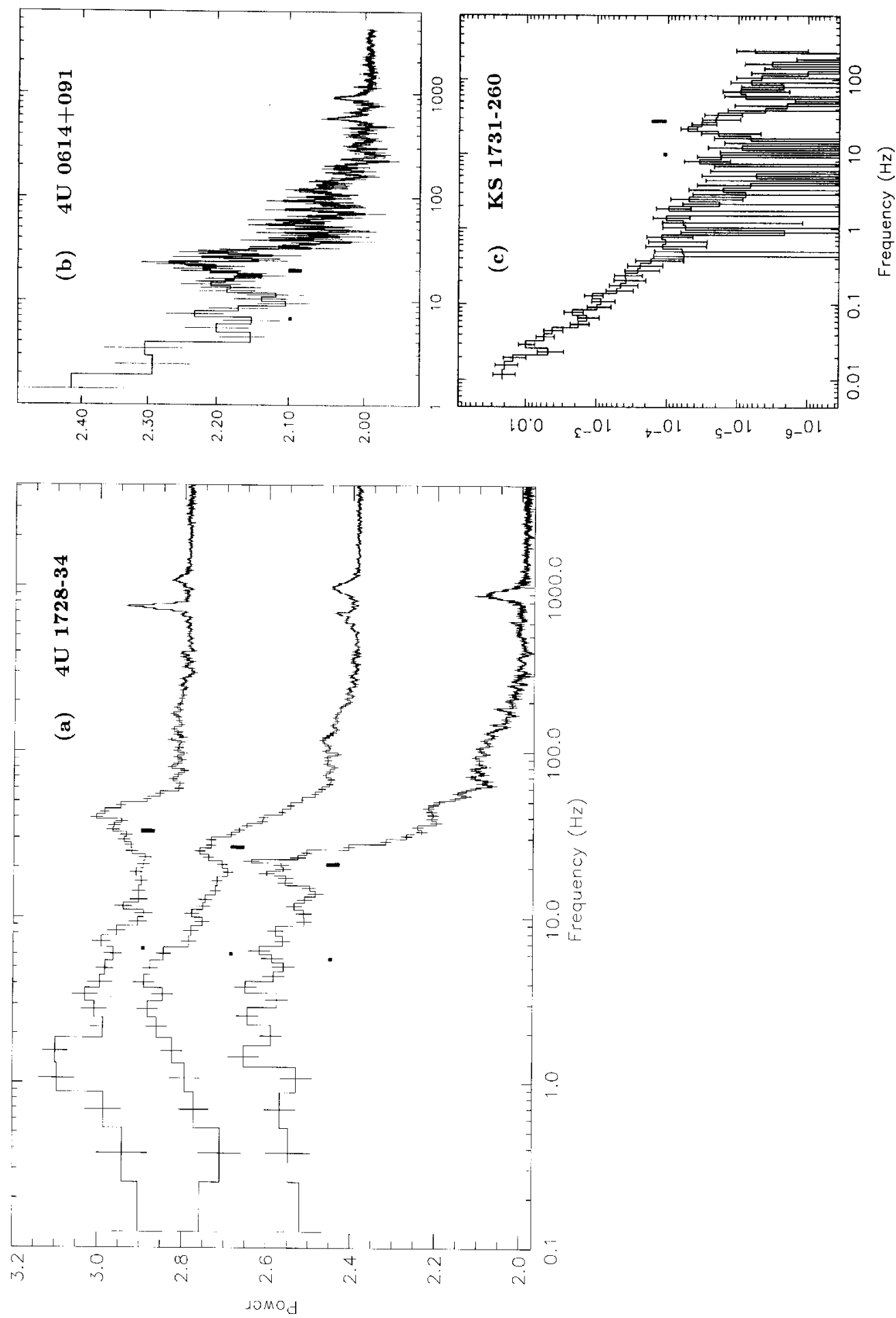

Fig. 1.- Power spectra of the RXTE light curves of 4U 1728-34 (panel $a$ ), 4U 0614+091 (panel $b$ ) and KS 1731260 (panel c) (after Strohmayer et al. 1996a, Ford et al. 1997 and Wijnands \& van der Klis 1997). The thick bars indicate the best precession frequency obtained from the model discussed in the text. The range of allowed precession frequencies for a variety of neutron star EOS and masses is marked by thick bars and filled squares. 